\title{
Visualization Terrain Data Using Cubic Ball Triangular Patches
}

\author{
Samsul Ariffin Bin Abdul Karim ${ }^{1, *}$ and Azizan Saaban ${ }^{2}$ \\ ${ }^{1}$ Fundamental and Applied Sciences Department and Centre for Smart Grid Energy Research \\ (CSMER), Institute of Autonomous System, Universiti Teknologi PETRONAS, Bandar Seri \\ Iskandar, 32610 Seri Iskandar, Perak DR, Malaysia. \\ ${ }^{2}$ College of Arts and Sciences, Universiti Utara Malaysia, 06010 UUM Sintok, Kedah, Malaysia.
}

\begin{abstract}
Scattered data technique is important to visualize the geometrical images of the surface data especially for terrain, earthquake, geochemical distribution, rainfall etc. The main objective of this study is to visualize the terrain data by using cubic Ball triangular patches. First step, the terrain data is triangulated by using Delaunay triangulation. Then partial derivative will be estimated at the data points. Sufficient condition for $\mathrm{C}^{1}$ continuity will be derived for each triangle. Finally, a convex combination comprising three rational local scheme is used to construct the surface. The scheme is tested to visualize the terrain data collected at central region of Malaysia.
\end{abstract}

\section{Introduction}

Scattered data interpolation is an active research in many years. This method is useful when the user wants to visualize certain types of real data sets. For instance, rainfall distribution and geochemical data. Besides that, scattered data also can be used for shape preserving interpolation such as to preserves the positivity of surface data. Some early studied includes Said and Rahmat [5] consider cubic Ball triangular for scattered data interpolation. Goodman and Said [3] discussed the method to construct a $\mathrm{C}^{1}$ surface by calculating three different inner Bezier ordinates for each triangle. The final surface is a convex combination from three local scheme. Piah et al. [4] has extended the work of [3] for positivity preserving. Their result is different that Chan and Ong [1] scheme. The main objective of this study is to improve the main stated result in Said and Rahmat [5] i.e. to derive the correct $C^{1}$ condition for scattered data interpolation.

\section{Cubic Ball triangular patches}

Said and Rahmat [5] has utilized the cubic Ball triangular for scattered data. They show that cubic Ball will produce $\mathrm{C}^{1}$ surface for the data obtained from some true function. But from the results, they claim that, their scheme gives same result as in Goodman and Said [3] except with lesser computation time. In this study, we will prove that the scheme presented

*Corresponding author: samsul_ariffin@utp.edu.my 
in Said and Rahmat [5] is not $\mathrm{C}^{1}$ at each of the adjacent triangles. We propose new sufficient condition for $\mathrm{C}^{1}$ continuity. We begin this section with some definition about cubic Ball triangular patch. Assume that, the barycentric coordinate $u, v, w$ on triangle $T$ with vertices $V_{1}, V_{2}$ and $V_{3}$ where $u+v+w=1$ and $u, v, w \geq 0$. From basic geometry, any point $V(x, y) \in R^{2}$ inside the triangle, can be defined as

$$
V=u V_{1}+v V_{2}+w V_{3}
$$

Figure 1 shows the distribution of the cubic Ball triangular bases on a triangular domain:

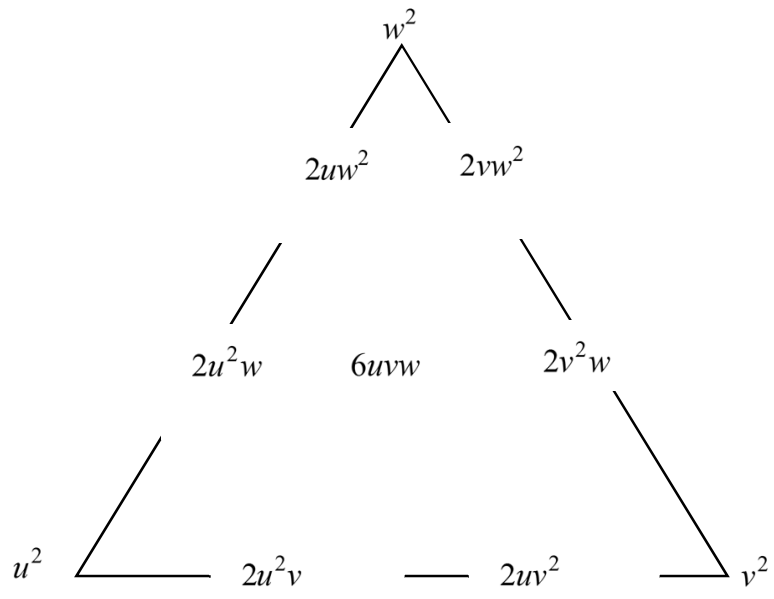

Fig. 1. Cubic Ball triangular bases.

Definition 1: The cubic Ball triangular patch with control points $b_{i j k}, i+j+k=3$ is defined as

$$
\begin{aligned}
P(u, v, w) & =u^{2} b_{300}+v^{2} b_{030}+w^{2} b_{003}+2 u^{2} v b_{210}+2 u^{2} w b_{201}+2 v^{2} u b_{120}+2 v^{2} w b_{021} \\
& +2 w^{2} u b_{102}+2 w^{2} v b_{021}+6 u v w b_{111}
\end{aligned}
$$

where $u+v+w=1$ and $b_{i j k} \in R^{3}, i+j+k=3$.

\section{Scattered data interpolation}

The scheme comprising convex combination of three local schemes $P_{1}, P_{2}$ and $P_{3}$ and is defined as

$$
P(u, v, w)=\frac{v w P_{1}+u w P_{2}+u v P_{3}}{v w_{1}+u w+u v}, u+v+w=1
$$


Local scheme $P_{i}, i=1,2,3$ is obtained by replacing $b_{111}$ in (2) with $b_{111}^{i}$ such that $C^{1}$ condition is satisfied only on the boundary $e_{i}$ of triangle.

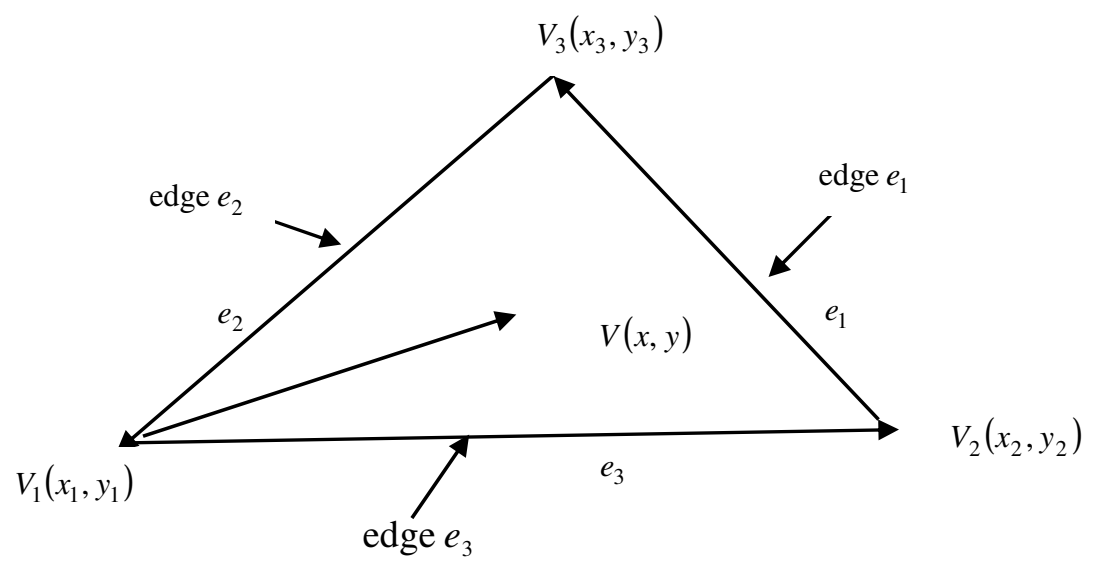

Fig. 2.Vertices and edges of the triangle.

Let the inward normal directions with the respective edges are given as $n_{1}, n_{2}$ and $n_{3}$ :

$$
n_{1}=-e_{3}+\frac{e_{3} \cdot e_{1}}{\left|e_{1}\right|^{2}}, n_{21}=-e_{1}+\frac{e_{1} \cdot e_{2}}{\left|e_{2}\right|^{2}} \text { and } n_{3}=-e_{2}+\frac{e_{2} \cdot e_{3}}{\left|e_{3}\right|^{2}}
$$

where "." is a scalar product. Firstly we will find the Ball ordinate $b_{111}^{1}$ by considering the normal derivatives along $n_{1}=(1,-1 / 2,-1 / 2)$ of local scheme $P_{1}$ i.e. on edge $V_{2} V_{3}$ such that $u=0, v+w=1$ :

$$
\begin{aligned}
D_{n_{3}} P_{1}= & (1)\left(2 b_{120} v^{2}+2 b_{102} w^{2}+6 v w b_{111}^{1}\right)-\left(\frac{1}{2}\right)\left(2 v b_{030}+4 v w b_{021}+2 b_{012} w^{2}\right) \\
& -\left(\frac{1}{2}\right)\left(2 w b_{003}+2 b_{021} v^{2}+4 b_{012} v w\right)
\end{aligned}
$$

which can be rearrange as

$$
D_{n_{3}} P_{1}=\left(2 b_{120}-b_{021}\right) v^{2}+\left(6 v w b_{111}^{1}-2 b_{012}-2 b_{021}\right) v w+\left(2 b_{102}-b_{012}\right) w^{2}+b_{030} v-b_{0303} w
$$

or

$$
D_{n_{3}} P_{1}=A v^{2}+B \mathrm{vw}+C w^{2}+b_{030} v-b_{0303} w
$$

For linearity,

$$
D_{n_{3}} P_{1}=A v^{2}+B \mathrm{vw}+C w^{2}
$$

where 


$$
\begin{aligned}
& A=2 b_{120}-b_{021}, \\
& B=6 v w b_{111}^{1}-2 b_{012}-2 b_{021}, \\
& C=2 b_{102}-b_{012}
\end{aligned}
$$

Theorem 1. The normal derivative in (5) must is linear on $e_{1}$ if and only if $B=A+C$.

\section{Proof.}

Substitute $B=A+C$ to (5) and $v+w=1$ since $u=0$ on edge $e_{1}$, we obtain :

$$
D_{n_{3}} P_{1}=A v^{2}+(A+C) \mathrm{vw}+C w^{2}=A v(v+w)+C w(v+w)=A v+C w=A(1-w)+C w
$$

which is linear.

From Theorem 1, the normal derivative will be linear on $e_{1}$ if

$$
6 v w b_{111}^{1}-2 b_{012}-2 b_{021}=2 b_{120}-b_{021}+2 b_{102}-b_{012}
$$

Thus

$$
b_{111}^{1}=\frac{1}{6}\left(2 b_{120}+2 b_{102}\right)+\frac{1}{12}\left(2 b_{021}+2 b_{012}\right)
$$

Similarly, we can obtain the other Ball ordinates i.e. $b_{111}^{2}$ and $b_{111}^{3}$ for local schemes $P_{2}$ and $P_{3}$, respectively.

Remak 2. In Said and Rahmat [5], they give the $C^{1}$ condition as

$$
b_{111}^{1}=\frac{1}{6}\left(2 b_{120}+2 b_{102}\right)+\frac{1}{12}\left(2 b_{021}-2 b_{012}\right)
$$

Based on Remark 2 and our derivation for $\mathrm{C}^{1}$ stated in (6), consequently we found that the scheme presented by Said and Rahmat [5] is not $\mathrm{C}^{1}$ and the current study is an improvement to the result stated in Said and Rahmat [5]. We can see this fact in the following section.

\section{Results and Discussion}

1. We test the proposed scattered data interpolation by using the new scheme based on cubic Ball triangular patches to visualize the digital elevation at Kalumpang Agricultural Station $\left(3^{0} 38^{\prime} \mathrm{N}, 101^{\circ} 34^{\prime} \mathrm{E}\right)$ in Selangor located about $90 \mathrm{~km}$ northeast of Kuala Lumpur, Malaysia as shown in Fig. 3. Meanwhile, Fig. 4 shows the results. Fig. 4(a) shows the Delaunay triangulation for all 165 data points. Fig. 4(b) shows the linear interpolant for the terrain data. Fig. 4(c) shows the example of surface reconstruction by using the proposed $\mathrm{C}^{1}$ scheme. Clearly the surface is smooth with visually pleasing. Meanwhile in Fig. 4(d) shows the surface when the $C^{1}$ condition in Said and Rahmat [5] is used. Based on Fig. 4(d), we can see that, Said and Rahmat [5] scheme is not $\mathrm{C}^{1}$ meanwhile the proposed scheme is this study is guaranteed to produce $\mathrm{C}^{1}$ surface everywhere. This is the significance of the present study i.e. has improved the result in [5]. 


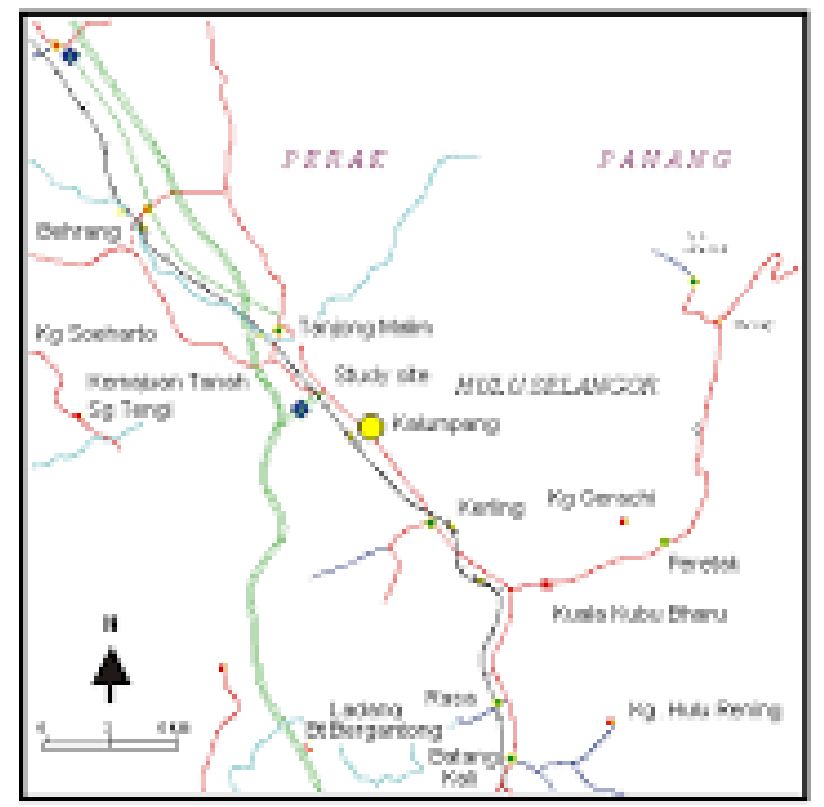

Fig. 3. Location of Kalumpang Agricultural Station in Malaysia.

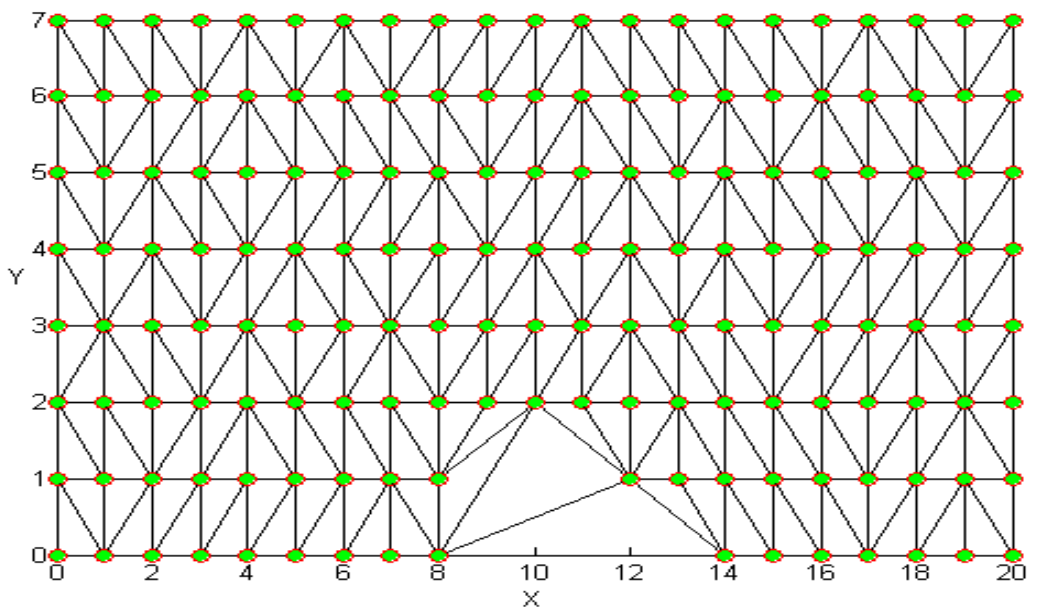

(a) 


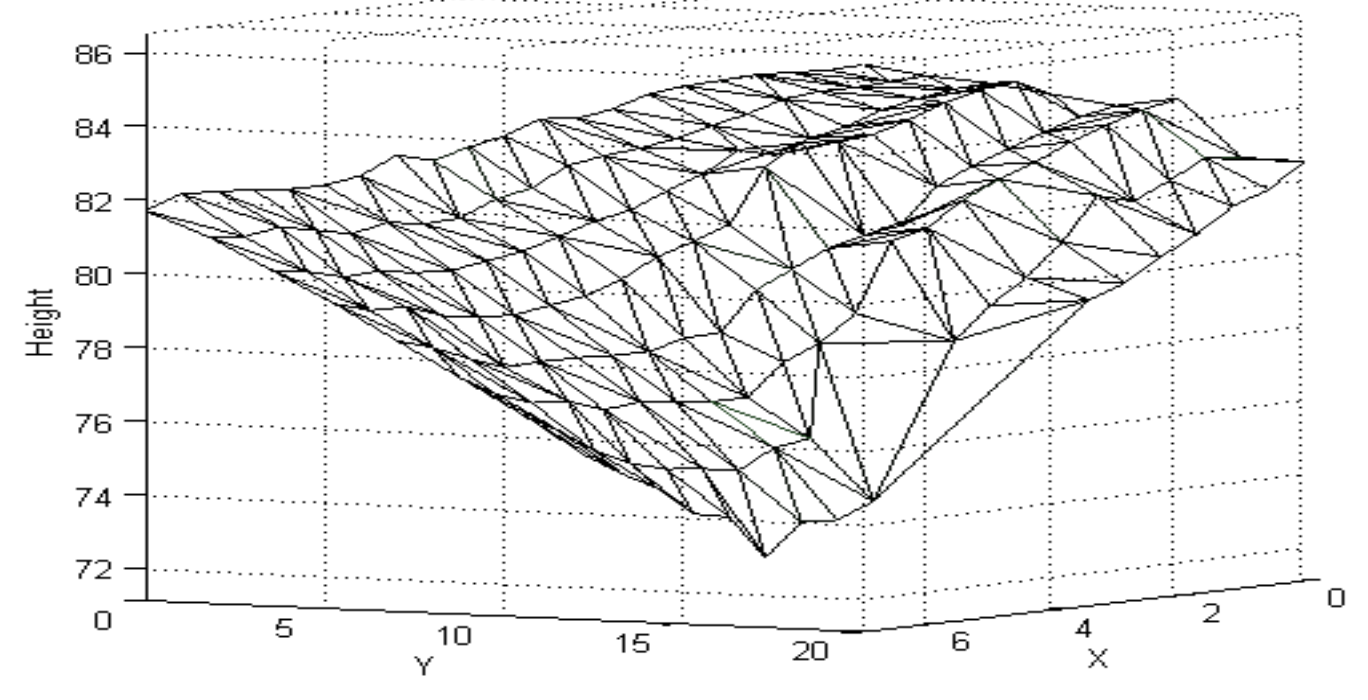

(b)

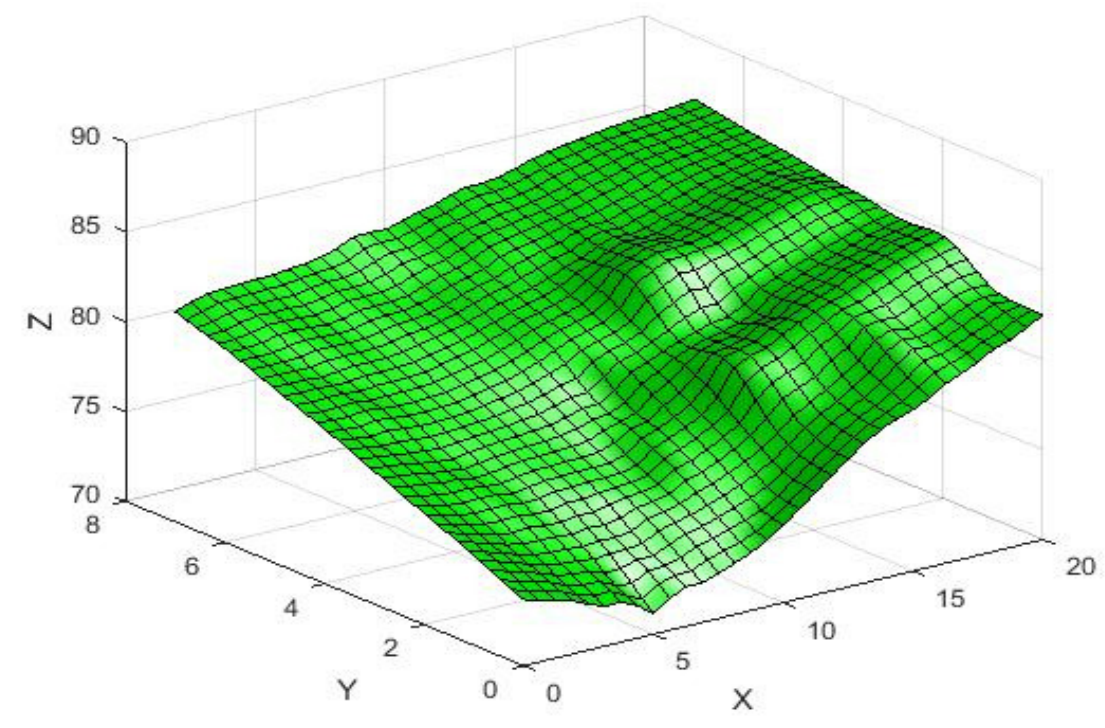

(c) 


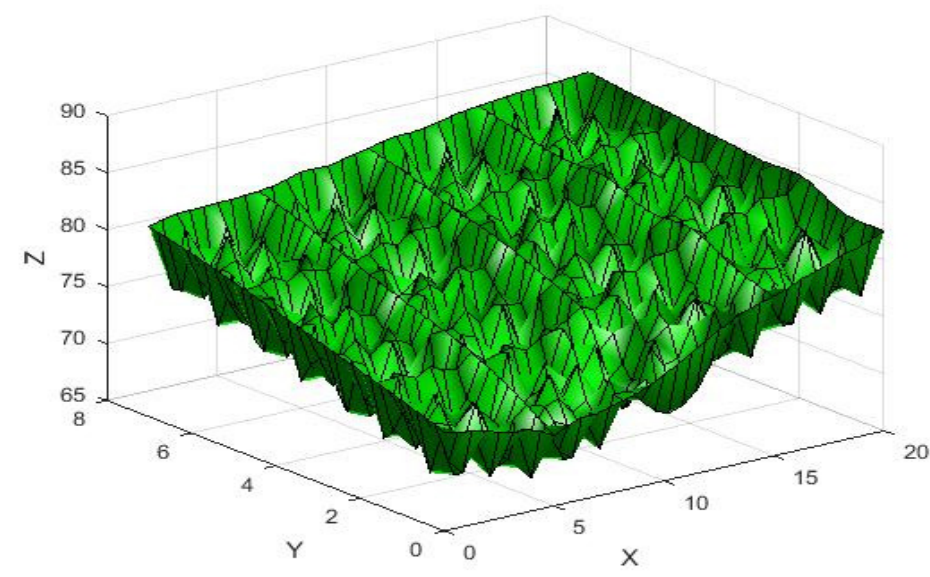

(d)

Fig. 4. Surface reconstruction for terrain data at Kalumpang Agricultural Station in Malaysia.

\section{Conclusion}

In this study, we derive a new $\mathrm{C}^{1}$ condition for cubic Ball triangles. The new condition is correct compared with the condition derived in Said and Rahmat [5]. As an application, we test the scheme to visualize the terrain data. From the results, we can see that, the cubic Ball triangular can reconstruct the surface with good approximation properties such as nice surface shape as well as $\mathrm{C}^{1}$ continuous along the boundaries. Future work will be applying the scheme for shape preserving interpolation and reconstruct the other types of surfaces, for instance, geo-chemical data and rainfall distribution as well as in medical imaging. An extension to the work of Foley and Optiz [3] is also possible.

Acknowledgement to Universiti Teknologi PETRONAS (UTP) for the financial support received in the form of research grant: YUTP: 0153AA-H24 including the MATLAB Software. This work were carried out when the second author is visiting the first author from 27th August 2018 until 30th August 2018.

\section{References}

1. E.S., Chan, and B.H., Ong, Journal of Computational and Applied Mathematics, vol. 136, pp. 135-147, (2001).

2. T.A. Foley, and K. Optiz, Hybrid cubic Bézier triangle patches. In T. Lyche and L.L. Schumaker (eds.), Mathematical Methods in Computer Aided Geometric Design II, Academic Press, 275-286, (1992).

3. T.N.T., Goodman, and H.B., Said, Communications in Applied Numerical Methods, vol. 7, pp. 479-485, (1991).

4. A.R.Mt., Piah, T.N.T., Goodman, and K., Unsworth, Positivity-preserving scattered data interpolation in Lecture Notes in Computer Sciences (LNCS) 3604, pp. 336-349, (2005).

5. H.B. Said, and R. W. Rahmat, Journal of Physical Sciences, Vol. 5, 89-101, (1994). 